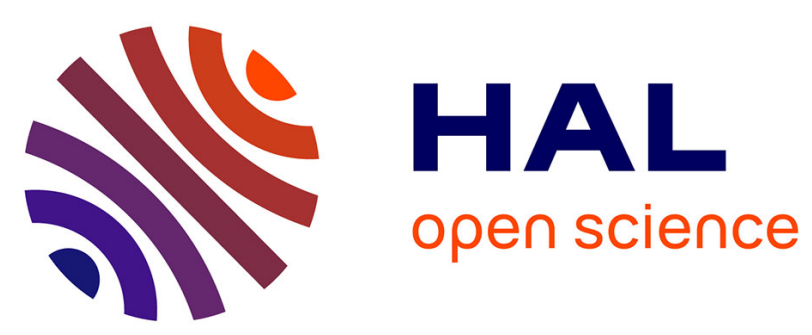

\title{
PRÉVISION DU BRUIT ET DIAGNOSTIC VIBROACOUSTIQUE D'UN SYSTÈME COMPOSÉ D'UN ROTOR SUR DEUX PALIERS À ROULEMENTS
}

Nacer Hamzaoui, C. Boisson, C. Lesueur

\section{To cite this version:}

Nacer Hamzaoui, C. Boisson, C. Lesueur. PRÉVISION DU BRUIT ET DIAGNOSTIC VIBROACOUSTIQUE D'UN SYSTÈME COMPOSÉ D'UN ROTOR SUR DEUX PALIERS À ROULEMENTS. Journal de Physique IV Proceedings, 1992, 02 (C1), pp.C1-467-C1-470. 10.1051/jp4:19921100 . jpa-00251054

HAL Id: jpa-00251054

https://hal.science/jpa-00251054

Submitted on 1 Jan 1992

HAL is a multi-disciplinary open access archive for the deposit and dissemination of scientific research documents, whether they are published or not. The documents may come from teaching and research institutions in France or abroad, or from public or private research centers.
L'archive ouverte pluridisciplinaire HAL, est destinée au dépôt et à la diffusion de documents scientifiques de niveau recherche, publiés ou non, émanant des établissements d'enseignement et de recherche français ou étrangers, des laboratoires publics ou privés. 


\title{
PREVISION DU BRUIT ET DIAGNOSTIC VIBROACOUSTIQUE D'UN SYSTÈME COMPOSÉ D'UN ROTOR SUR DEUX PALIERS À ROULEMENTS
}

\author{
N. HAMZAOUI, C. BOISSON et C. LESUEUR \\ Laboratoire Vibrations-Acoustique, Bât. 303, I.N.S.A., 20 avenue Albert Einstein, \\ F-69621 Villeurbanne cedex, France
}

\begin{abstract}
:
This work is part of a study for sound prediction and vibroacoustic diagnosis of machine. We present the first experimental and theoretical results obtained for a testing bench composed of three supports (motor, rotor on bearing and reductor) to simulate such experimental defects as mesalignment ,unbalancement, defective bearings and gears.These results concern more specifically the rotor on bearing support.The theoretical part is the calculation of pressure or acoustic power ,from structure vibratory velocity measurement . The basis of the modelisation is a point source distribution on the structure area, located on a rigid ground, radiating in a semi-anechoic medium. The experimental part is the simulation of several real defects (angular and parallel mesalignment and unbalancement) and the measurement of the resulting acoustic power using intensimetry techniques and acoustic pressure. Theory experiment comparison are presented..

I - Introduction

L'objectif est donc d'associer les connaissances sur le diagnostic vibratoire détectant les causes principales dûes à des défauts internes, à une partie acoustique incluant des critères de tri fréquentiels et spatiaux. Pour atteindre cet objectif, nous procédons par graduation, à partir de modélisations théoriques confrontées à des expériences, afin de maîtriser tous les paramètres liés aux difficultés que l'on peut rencontrer dans ce type de problème.

Dans cet article, nous analysons une modélisation thérique permettant la prévision des niveaux de pression ou de puissance acoustique à partir de mesures des vitesses vibratoires de la machine. Nous présentons aussi quelques résultats expérimentaux sur un système moteur-arbre sur paliers à billes permettant des configurations de défauts classiques.

II - Approche théorique

Le problème est de calculer la pression acoustique en milieu extérieur, à partir de mesures vibratoires sur la surface d'une structure placée au dessus d'un sol rigide. L'élément de base de ce problème est constitué par la formulation intégrale, reposant sur le théorème de Green, de la pression acoustique :
\end{abstract}

$$
P(M)=\iint_{S}\left(P\left(M_{0}\right) \frac{\partial G}{\partial n_{M_{0}}}\left(M, M_{0}\right)-\frac{\partial P}{\partial n_{M_{0}}}\left(M_{0}\right) G\left(M, M_{0}\right)\right) d S_{M_{0}}
$$

La fonction de Green respectant les conditions aux limites de ce problème, est determinée grace au principe de la source image :

avec

$$
G\left(M, M_{0}\right)=G_{1}\left(M, M_{0}^{\prime}\right)+G_{2}\left(M, M_{0}^{\prime \prime}\right)=\frac{e^{-j k r_{1}}}{4 \pi r_{1}}+\frac{e^{-j k r_{2}}}{4 \pi r_{2}}
$$

$$
\begin{aligned}
& r_{1}=\left(\left(x_{M}-x_{M_{0}}\right)^{2}+\left(y_{M}-y_{M_{0}}\right)^{2}+\left(z_{M}-z_{M_{0}}\right)^{2}\right)^{1 / 2} \\
& r_{2}=\left(\left(x_{M}-x_{M_{0}}\right)^{2}+\left(y_{M}-y_{M_{0}}\right)^{2}+\left(z_{M}+z_{M_{0}}\right)^{2}\right)^{1 / 2}
\end{aligned}
$$


Ce problème est résolu à partir d'une discrétisation de la surface de la structure en utilisant la méthode de collocation.

Pour une structure massique telle qu'une machine tournante, nous proposons une approche basée sur une distribution monopolaire de la surface vibratoire de la machine dont la pression acoustique rayonnée s'obtient par :

$$
\widetilde{P}(M)=j \rho_{0} \omega \sum_{i=1}^{N_{e}}|V| e^{i}\left(\varphi_{\text {ref }}-\varphi_{i}\right) G\left(M, M_{i}\right) \Delta S_{i}
$$

Chaque élément de surface $\Delta S_{i}$, représentant un monopôle, est caractérisé par le module de sa vitesse vibratoire $|V| \mathfrak{f}$ et la phase $\left(\varphi_{\text {ref }}-\varphi_{i}\right)$ relevée en utilisant un point de référence fixe pendant la durée d'acquisition des Ne points de mesures vibratoires.

A partir d'une formulation exacte de l'intensité acoustique active, le calcul de la puissance acoustique est effectué par :

$$
\begin{aligned}
W \approx \sum_{i=1}^{N} \vec{I}_{i} \vec{n}_{i} \Delta \Gamma_{i} \quad \text { avec } \quad \vec{I}_{i}=\frac{1}{2} R_{e}\left(P_{i} \cdot \frac{j}{\rho_{0} \omega}\left(\begin{array}{l}
\partial P_{i} / \partial x^{*} \\
\partial P_{i} / \partial y^{*} \\
\partial P_{i} / \partial z^{*}
\end{array}\right)\right. \\
* \text { : conjugué } \quad \Gamma \approx \sum_{i=1}^{N} \Delta \Gamma_{i} \text { : surface de contrôle }
\end{aligned}
$$

\section{III - Mesures sur banc d'essai}

L'étude expérimentale est réalisée sur un banc d'essai constitué de trois platines (moteur, rotor sur paliers, réducteur) et permettant des configurations simulant des défauts de balourd, de mésalignement et de roulement défectueux.

Les mesures consacrées à la platine "rotor sur palier", avec isolation acoustique des deux autres platines, concernent :

1 - Les vitesses vibratoires en plusieurs points de la platine sans configuration de défaut, afin de calculer $\widetilde{\mathbf{P}}(\mathbf{M})$ (Cf. (5)).

2 - La pression et la puissance acoustique par intensimétrie avec des simulations de défauts de balourd et de mésalignement, et sans défaut.

Nous signalons que l'ensemble du banc d'essai est entouré de plaques de mousse alvéolées formant ainsi un champ acoustique calme, et que les mesures acoustiques sont réalisées grâce à un système de bras mobile suivant deux directions perpendiculaires, entièrement piloté par ordinateur.

\section{IV - Résultats}

Confrontée à la mesure expérimentale, la pression $\widetilde{\mathrm{P}}(\mathrm{M})$ (Cf. expression (5)) est entachée d'une erreur $\Delta \mathrm{P}$ qui est fonction :

1 - Du type de champ acoustique régnant autour de la machine.

2 - Du maillage vibratoire.

3 - Des variations de phase $\left(\varphi_{\text {ref }}-\varphi_{i}\right)$.

4 - Des incertitudes de mesures.

5 - De l'annulation de l'effet dipolaire.

Mis à part l'effet dipolaire, nous avons analysé à partir de tests de sensibilité théorique, l'effet des autres parametres sur le calcul de la pression acoustique.L'effet de variation de la phase, permettant d'accéder à la vitesse vibratoire complexe est le plus influent et imprévisible pour le calcul de la pression acoustique ; la nature du champ a aussi une influence non négligeable.

En l'absence de défaut, les niveaux vibratoires et acoustiques mesurés sont très faibles et les variations de phase, au cours du temps, entre deux points fixes sont très importantes. Les diverses confrontations entre la théorie et l'expérience, présentent un aspect reproductible du point de vue des spectres, mais quantitativement les comparaisons restent insatisfaisantes en dehors de la fréquence de rotation et de ses harmoniques. 
Le mésalignement angulaire au niveau d'un palier augmente le niveau global du bruit $(+10 \mathrm{~dB}$ en puissance acoustique, $+20 \mathrm{~dB}$ en pression au voisinage du palier mésaligné $\left(0.8^{\circ}\right)$ ). Le balourd provoque une augmentation du niveau de pression à la fréquence de rotation et à l'harmonique 2 de cette dernière.

\section{V -Conclusions}

Au dessus de $200 \mathrm{hz}$, les valeurs maximales du spectre sont en général bien appréhendées par le calcul.Il faut cependant améliorer la discrétisation spatiale en donnant un poids plus important à des surfaces petites qui fournissent beaucoup de bruit : c'est le cas des paliers.La parfaite réalisation du banc d'essai montre bien qu'en l'absence de défauts, les niveaux vibratoires et acoustiques sont faibles et le bruit de fond de l'appareillage et du milieu acoustique devient prépondérant.La relation entre défauts et génération de bruit est donc bien mise en évidence et devrait permettre d'établir des critères de qualité acoustique d'une machine.

Ce travail rentre dans le cadre d'un projet en collaboration avec II.N.R.S de NANCY et la Societé CAMPAGNA et VARENNE de VIENNE, finance par le Ministère de la Recherche et de la Technologie et le Ministère du Travail ( FACT " Construire silencieux") que l'on remercie vivement.

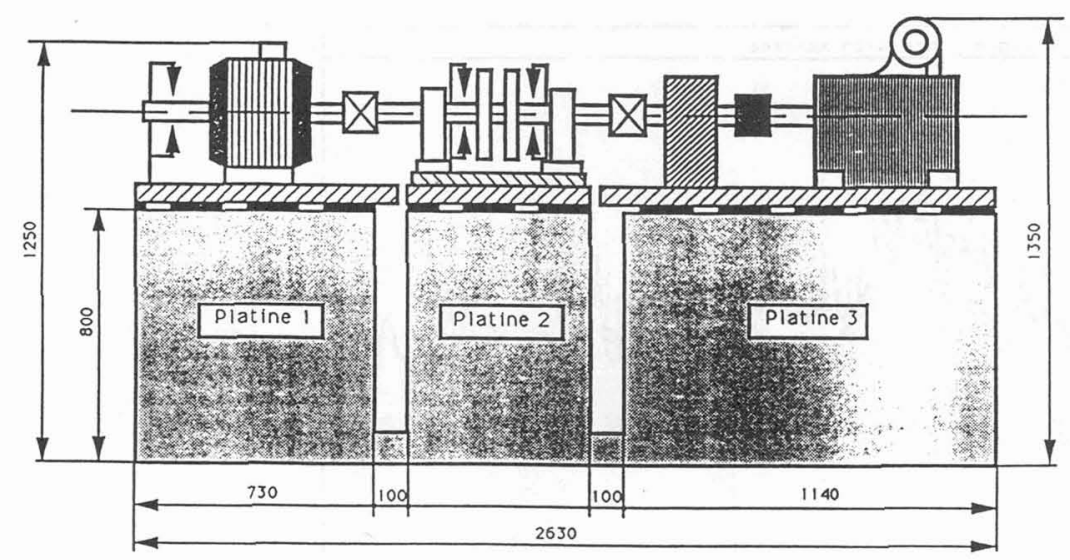

FIGURE 1.: Banc d'essai.

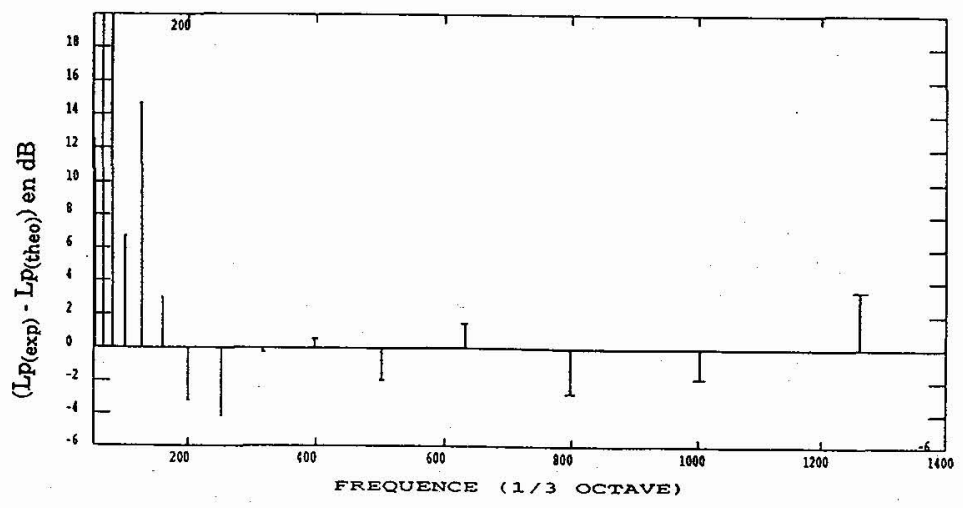

Eiqure 2 Différence entre pression mesurée et pression calculée au point $(0.35,0.17,0.23)$ (configuration avec défaut) 

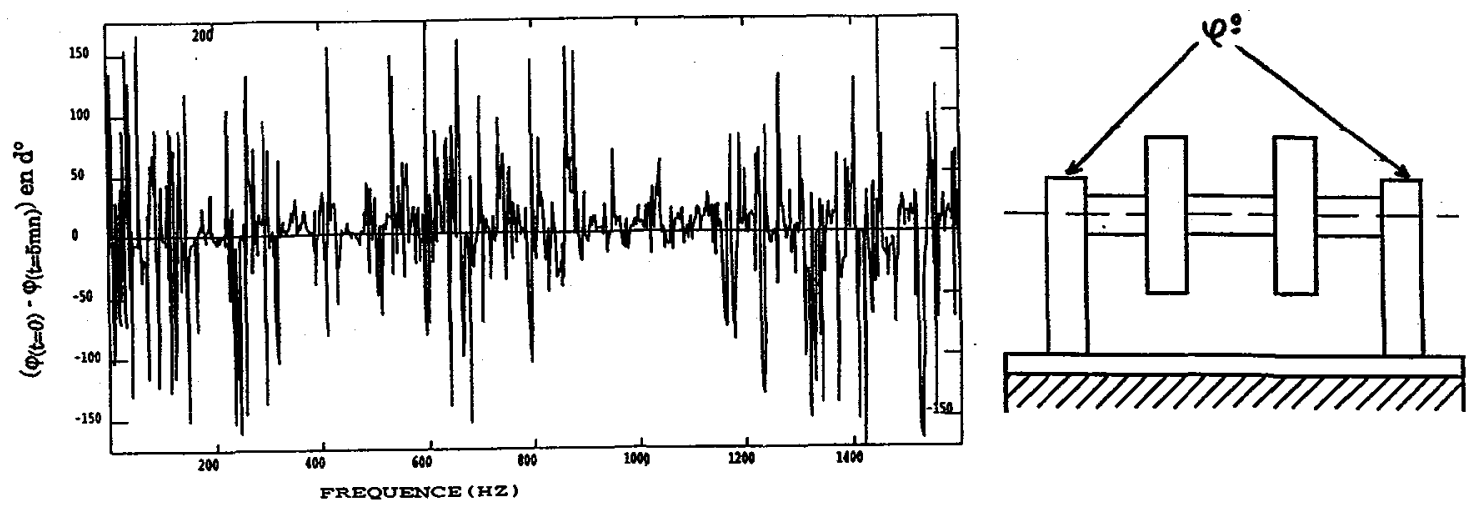

Figure 3 : Variations expérimentale de la phase

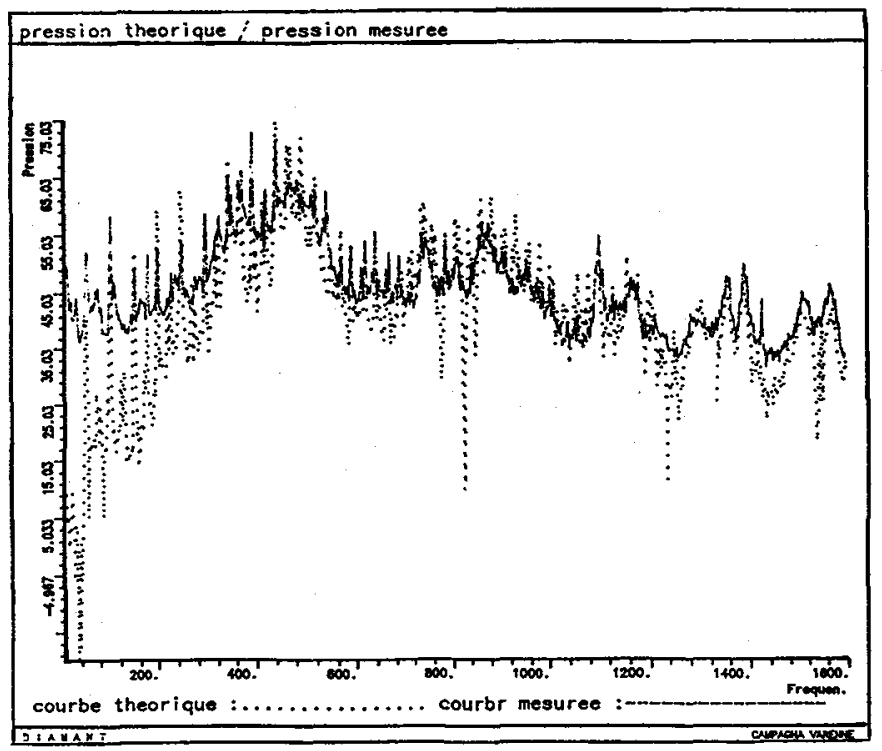

Figure 4 : Niveau de pression acoustique calculé et mesuré .

\section{Bibliographie:}

(1) J.P. THOME, N. HAMZAOUI, C. MILLARD , 'Methodologie d'approche industrielle pour l'identification et la caractérisation des sources de bruit d'une machine tournante .' Publication du L.M.A $n^{\circ} 126$ (Sept. 91), rencontre scientifique du cinquentenaire $(345,369)$.

(2) C. LESUEUR, ' Rayonnement acoustique des structures vibroacoustiques , interactions fluide structure'. Editions Eyrolles 66,1988 .

(3) N. HAMZAOUI. 'Etude du champ acoustique de plaques par intensimétrie. Contribution à la définition d'une méthodologie de mesure de la puissance acoustique.' Thèse INSA LYON 1985.

(4) C. REMILLAT ,B. BARTHOU 'Diagnostic vibroacoustique d'un rotor'. Rapport de projet de fin d'etude .G.M.C INSA LYON 1991. 